\title{
Health research in the state of Odisha, India: a 10-year bibliometric analysis (2011-2021)
}

\author{
Jaya Singh Kshatri, Parmeshwar Satpathy, Sumita Sharma, Trilochan Bhoi, Smruti \\ Prakash Mishra, Soumya Swaroop Sahoo*
}

Correspondence: Dr. Soumya Swaroop Sahoo, Ass. Prof., Department of Community Medicine, AIIMS, Bhatinda, Email: swaroop.drsoumya@gmail.com

\section{ABSTRACT:}

Background: Bibliometric analyses are an important tool for evaluating health research outputs in terms of their distribution, trends, actors, focus, and funding sources. The transition from Millennium to sustainable development goals have led to a gradual shift in health policy and possibly the research priorities of low-income settings in the Empowered Action Group (EAG) states, lagging in socio-economic and health parameters, also ranking low on innovations and research. This study was aimed to describe the recent trends, quantity, type, focus and sources of health-related research in the EAG state of Odisha, India.

Materials and Methods: Peer reviewed published original research articles which were related to human health, published between 1st Jan 2011 and 31st Dec 2020 and where the study population were residents of Odisha, or study site was in Odisha, exclusively or partially were analyzed. Publication characteristics were tabulated, including title, journal name, open access, date of publication, number of authors, designation of the authors, number of institutes involved, name of institute of first author. Details of study setting, study site, ethical clearance, funding source were also collected.

Results: The study identified 2285 articles from database searches and included 666 articles after screening for the bibliometric analysis. Most of the manuscripts had between 3-6 authors (43.5\%). Two institutes from the state, ICMR-RMRC and KIIT, together contributed to over $22.4 \%$ of the published manuscripts as lead authors. Nearly $45.9 \%$ studies were community based while $45.3 \%$ were hospital based. While most of the published work was on infectious diseases, the proportion came down with time between 2011 and 2020. An overwhelming majority of studies were observational in nature and less than $10 \%$ were experimental in design.

Conclusions: The analysis shows a substantial increase in the number of publications in this decade. Priority setting of health care problems, increased funding and capacity building can give a much-necessitated impetus to more quality and evidence-based research for aiding policy implementation and improvement of overall health of Odisha.

Keywords: Bibliometric analysis, health research, publication trends 


\section{Background and rationale:}

The definition of health research is broad and not just includes biomedical research, epidemiological studies and health services research but also studies on behavioral, social, and economic factors affecting health. The value of research towards advancing innovations and improving patient care, healthcare services, systems, policies, and overall wellbeing of the communities is well recognized. (1)

The quality, quantity and impact of health research is an indirect measure of the maturity of the health care systems and reflects the national policy priorities of a country. Evidently, there are wide variations in health research outputs between and even within countries. In a large and diverse country such as India, with one-sixth of humanity and a large proportion of global disease burden, the health research support systems and consequently the research output has generally been inadequate.(2) Historically, the meagre resources allocated to health research were concentrated on control of communicable diseases, reduction in fertility, maternal and child health, nutritional disorders and other health challenges of the $20^{\text {th }}$ century.(2) The health research output is not equitably distributed across the country and has been concentrated in a few institutions with only $4 \%$ elite institutions contributing to over $40 \%$ of research output from the country. $(3,4)$ Additionally, regional disparities across the country persist with eight socioeconomically backward states of Bihar, Chhattisgarh, Jharkhand, Madhya Pradesh, Odisha, Rajasthan, Uttaranchal and Uttar Pradesh, referred to as the Empowered Action Group (EAG) states, lagging in socio-economic and health parameters, also ranking low on innovations and research. $(2,3)$

A part of this group, the state of Odisha on the eastern coast of India, with a significant population of indigenous tribes, has been ranked in the bottom 3 in the country using a composite health index comprising of health outcomes, governance, information, key inputs, and processes.(5) However, sustained efforts to address these challenges and ensure equity in healthcare have seen significant improvements in the state during the past decade.(6) This period has also seen an increased focus on public health, with establishment of a dedicated cadre of health professionals in the public health system and increase in public health training and research institutions in the state. The transition from Millennium to sustainable development goals have led to a gradual shift in health policy and possibly the research priorities of low-income settings such as Odisha.

Bibliometric analyses are an important tool for evaluating health research outputs in terms of their distribution, trends, actors, focus, and funding sources. This would also be useful in identifying the gaps in research ecosystems and addressing them. To the best of our knowledge, there is no data on the quantification of health research outputs or changing trends of health research priorities among the EAG states such as Odisha in India. In this study we aim to describe the recent trends, quantity, type, focus and sources of health-related research in the state of Odisha, India between 2011 and 2020.(7)(8,9)(6,10)

\section{Methodology:}

This was a prospectively registered bibliometric analysis of published literature from the state of Odisha. A comprehensive systematic search was performed on January 1, 2021, in the following electronic databases: Medline (via Pubmed), Embase (via Ovid), PsycINFO (via Ovid) and CINAHL (via EBSCOHost). The databases were searched for studies that had a mention of the name of the state and its variations as well as the name of all the districts and 
major cities in the state within their abstracts and titles. The detailed search strategy is provided in Appendix 1.

Following de-duplication, articles were screened using Rayyan software by two independent review authors based on their Title/Abstract. (11) We included Peer reviewed published original research articles which were related to human health, published between 1st Jan 2011 and 31st Dec 2020 and where the study population were residents of Odisha, or study site was in Odisha, exclusively or partially. We excluded studies from the field of environmental science, physical sciences, botany or veterinary sciences, laboratory, biological or chemical research not related to human health directly. We also excluded conference abstracts or presentations, protocols, books/book chapters, preprints, reviews- narrative or systematic, letters/news articles/opinions/commentaries, and studies carried out at the national level- with none of the objectives involving the state or population of Odisha in full or in part.

Discrepancies were discussed and resolved by consensus. In case of disagreement, a third author made the decision. Full texts were retrieved and reviewed for eligibility subsequently. Full-text review followed the same method as Title/abstract screening in case of disagreement.

Through an iterative process, a predefined data extraction sheet was developed to capture all relevant aspects of the included studies. Publication characteristics were tabulated, including title, journal name, open access, date of publication, number of authors, designation of the authors - classified as junior faculty (Assistant professor)/senior faculty (Associate Professor and above) resident/scientist/others, number of institutes involved, name of institute of $1^{\text {st }}$ author. Details of study setting, study site, ethical clearance, funding source were collected.

The studies were further classified as per their study domains using the ICD-11 framework. (12) Similarly, study design was classified as per SAGE Research methods framework. (13) Descriptive statistics and cross tabulations were performed using "R software ver. 4.0.3". (14) Frequencies were used to describe outputs over time, institutes, authors, study types/setting/funding, and examine outputs in different disease domain over time, geographical distribution of studies/institutions, collaborations. The retrieved literature was exported to VOS viewer program to create network visualization maps.(15)

\section{$\underline{\text { Results: }}$}

The study identified 2285 articles in total from database searches and finally included 666 articles after screening for the bibliometric analysis as shown in Figure-1. 


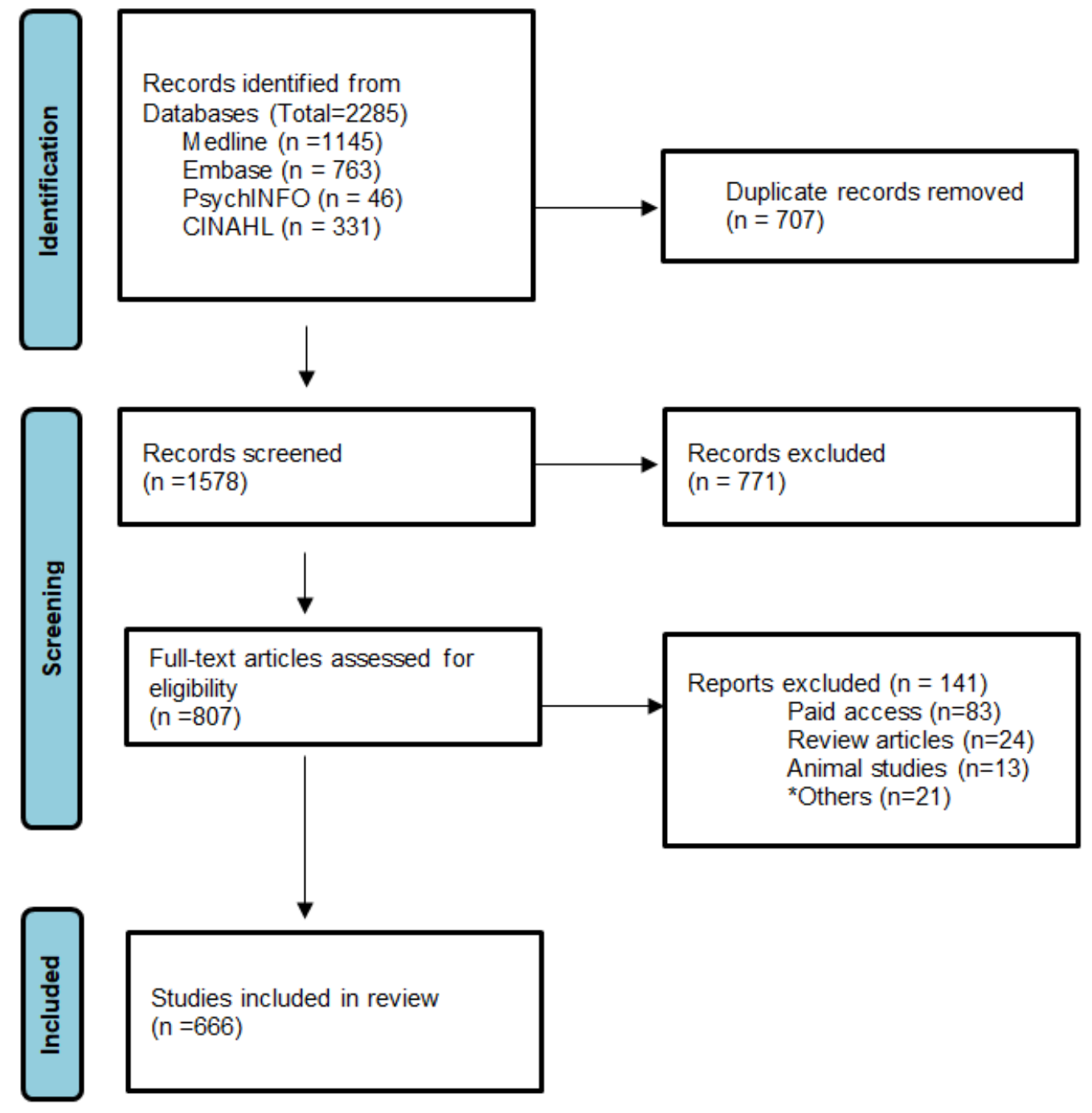

*Others includes Duplicates, Studies outside Odisha, Letter to Editor, Conference Abstract, Medicinal plant studies

Figure 1: PRISMA flow chart of bibliometric review

Most of the manuscripts had between 3-6 authors (43.5\%) while 28.0\% of the manuscripts had either 2 or 3 authors and 22.6\% articles had between 6 and 15 authors. Only 3.3\% manuscripts had a single author and $2.4 \%$ had more than 15 authors. Two institutes from the state, ICMR-RMRC and KIIT, together contributed to over $22.4 \%$ of the published manuscripts as lead authors. The contribution of other institutes was lesser, as shown in Figure-2 below. 


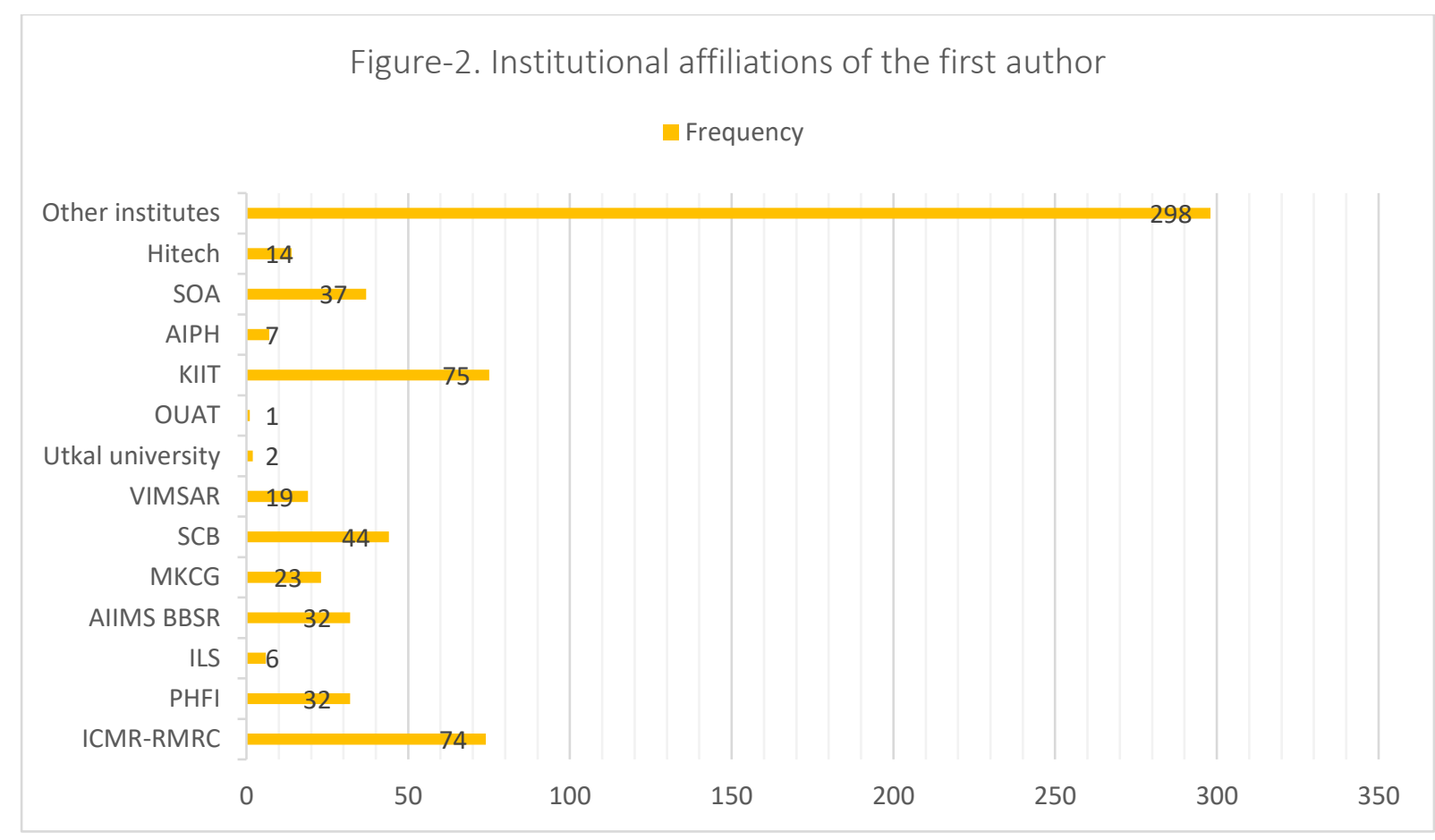

Footnote: $(\mathrm{SOA}=$ Sikshya 'O' Anusandhaan University, AIPH= Asian Institute of Public Health, KIIT= Kalinga Institute of Industrial Technology, OUAT= Odisha University of Agriculture \& Technology, VIMSAR= Veer Surendra Sai Institute of Medical Sciences And Research, SCB= Srirama Chandra Bhanja Medical College and Hospital, MKCG= Maharaja Krishna Chandra Gajapati Medical College \& Hospital, AIIMS BBSR= All India Institute of Medical Sciences Bhubaneswar, ILS= Institute of Life Sciences, PHFI= Public Health Foundation of India, ICMR-RMRC = Indian Council of Medical Research- Regional Medical Research Center)

While $45.9 \%$ studies were community based, $45.3 \%$ were in hospitals and $2.1 \%$ were in both these settings. The rest were primarily laboratory-based studies. The overall quantity research publications per year increased steadily from 22 in 2011 to 99 in 2020 . While most of the published work was on infectious diseases, the proportion came down with time between 2011 and 2020. The domain specific trends with time are provided in Figure-3 below. An overwhelming majority of studies were observational in nature and less than $10 \%$ were experimental in design. Table- 1 shows the distribution of study designs. 
Figure-3. Trends of research publications in Odisha between 2011 and 2020

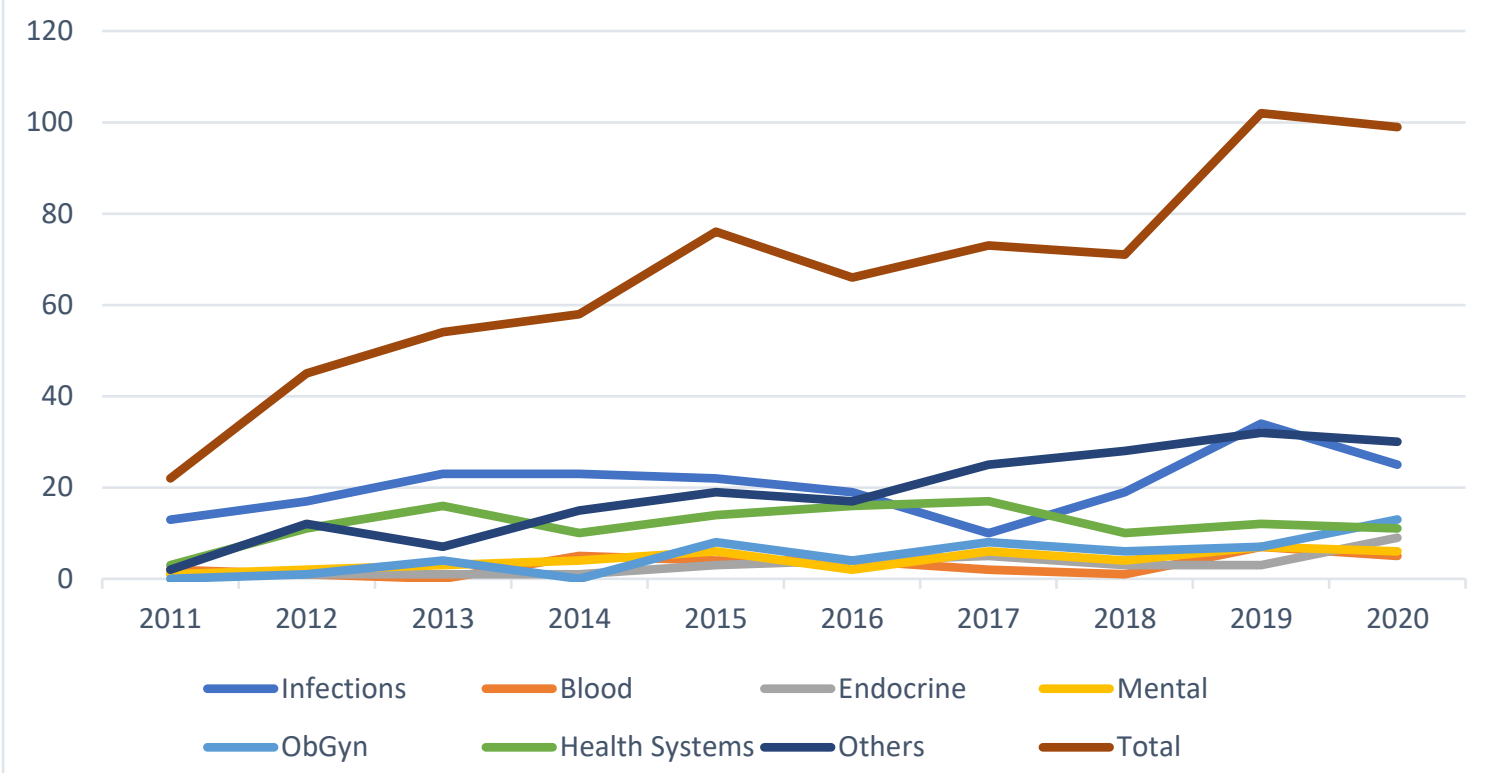

Table-1. Study designs and the study population studies from Odisha between 2011-2020

\begin{tabular}{|c|c|c|c|c|c|}
\hline \multirow{2}{*}{ Study design } & \multicolumn{4}{|c|}{ Study population } & \multirow{2}{*}{ Total } \\
\cline { 2 - 5 } & Urban & Rural & Both & Others & \\
\hline Case study & $10(4.9 \%)$ & $9(4.4 \%)$ & $5(2.5 \%)$ & $5(8.9 \%)$ & $29(4.4 \%)$ \\
\hline Case series & $7(3.4 \%)$ & $0(0.0 \%)$ & $6(3.0 \%)$ & $0(0.0 \%)$ & $13(2.0 \%)$ \\
\hline Ecological study & $0(0.0 \%)$ & $0(0.0 \%)$ & $1(0.5 \%)$ & $2(3.6 \%)$ & $3(0.5 \%)$ \\
\hline Qualitative study & $4(2.0 \%)$ & $28(13.7 \%)$ & $10(5.0 \%)$ & $4(7.1 \%)$ & $46(6.9 \%)$ \\
\hline Cross sectional & 145 & 115 & 149 & $38(67.9 \%)$ & 447 \\
$(71.1 \%)$ & $(56.4 \%)$ & $(73.8 \%)$ & $167.1 \%)$ \\
\hline Case control & $12(5.9 \%)$ & $3(1.5 \%)$ & $11(5.4 \%)$ & $1(1.8 \%)$ & $27(4.1 \%)$ \\
\hline Cohort & $10(4.9 \%)$ & $16(7.8 \%)$ & $15(7.4 \%)$ & $1(1.8 \%)$ & $42(6.3 \%)$ \\
\hline Quasi experimental & $4(2.0 \%)$ & $4(2.0 \%)$ & $0(0.0 \%)$ & $3(5.4 \%)$ & $11(1.7 \%)$ \\
\hline Cluster/Community & $2(1.0 \%)$ & $16(7.8 \%)$ & $3(1.5 \%)$ & $0(0.0 \%)$ & $21(3.2 \%)$ \\
\hline trials & $10(4.9 \%)$ & $13(6.4 \%)$ & $2(1.0 \%)$ & $2(3.6 \%)$ & $27(4.1 \%)$ \\
\hline RCT & 204 & 204 & 202 & 56 & 666 \\
\hline Total & $(100.0 \%)$ & $(100.0 \%)$ & $(100.0 \%)$ & $(100.0 \%)$ & $(100.0 \%)$ \\
\hline
\end{tabular}

Most of the studies were carried out in the central districts (43.2\%) followed by southern districts $(10.3 \%)$ and northern districts $(9.5 \%)$. While $24.5 \%$ studies were across the regions of the state, $12.5 \%$ were between multiple states.

Among the 213 articles who had declared their sources of funding, most of them were funded by foreign entities (42.2\%). ICMR funded research were $22.1 \%$ and DBT/DST funded $7.0 \%$ of the studies. The declarations on ethical clearance and funding details are provided in table2 below. 
Table-2. Declarations made in the manuscripts in Odisha between 2011-2020

\begin{tabular}{|l|l|l|}
\hline Declaration & Declared & Not Declared \\
\hline Ethical Clearance & $424(63.7 \%)$ & $242(36.3 \%)$ \\
\hline Funding status & $331(49.7 \%)$ & $335(50.3 \%)$ \\
\hline Sources of funding & $213(64.3 \%)$ & $118(35.7 \%)$ \\
\hline
\end{tabular}

There was significant clustering of major groups of authors with little collaborations between these groups as shown in Figure-4. The changing trend of author groups collaborations is shown in figure-5.

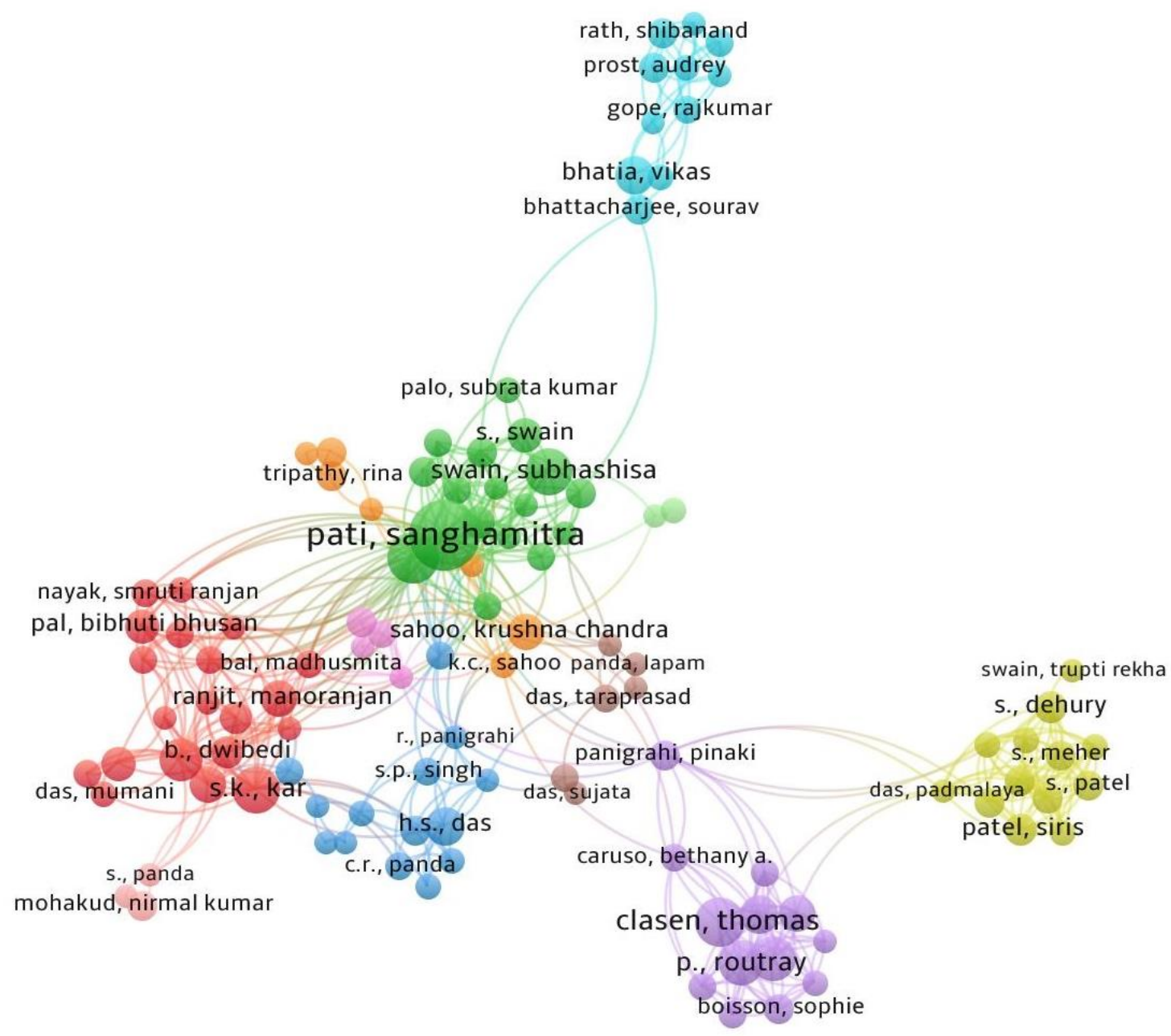

Figure-4. Major contributors to published papers (diameter of the circles) groups of authors (colors) and the networks for collaborations (lines) 


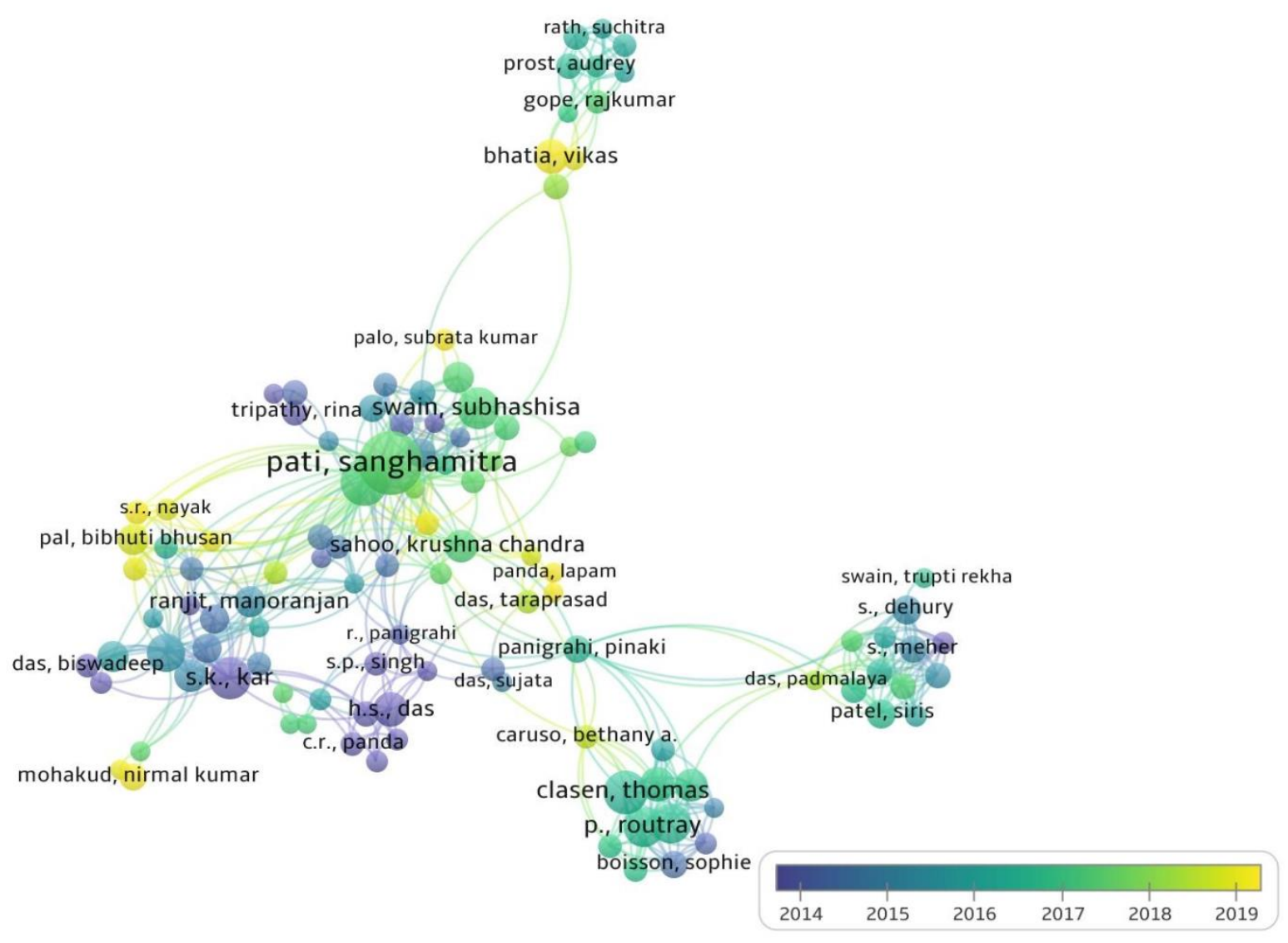

Figure-5. Changing trends of author collaborations between 2011-2020.

\section{Discussion:}

This comprehensive bibliometric analysis was carried out to describe the state of health research in the state of Odisha between 2011-2020. To the best of our knowledge this study is the first such study that evaluates the trend of published health research from the region.

The quantity of published heath literature from the State of Odisha has witnessed a significant and steady increase from 2011-2020 with maximum number of articles published in 2019 . Annual publications were relatively stagnant at less than 50 per year in the decade preceding the study period, however this has more than doubled over the past decade.(2) However, the overall output in terms of quantity is still very low when compared to the rest of the country and is comparable to other EAG states such as Bihar. $(2,16)$ This increase in research output may be attributed to multiple factors such as This rise in the number of publications can be attributed mostly to the increased number of public health academic institutions, increased funding sources and newer regulatory guidelines reinforcing academic research as a stringent criterion for faculty promotions. 
This increase in quantity of publications is associated with a change in the focus areas of health research in the state. Over time, research into social, environmental, and other determinants of health have increased in proportion. While most of the articles between 2011 and 2020 were related to Infectious disease domain, there is a gradual increase in research into diseases of the blood or blood-forming organs, endocrine system, nutritional or metabolic diseases, mental health, behavioral or neurodevelopmental disorders, etc. Similar diversification is a feature of the changing trends of focus areas for health research across India over the past decade.(3) With an increase in burden of non-communicable diseases commensurate with a decrease in burden of infectious and maternal/child health issues, the priorities of both the health systems and health research in India seem to be changing in the $21^{\text {st }}$ century.

Nearly two-thirds of the studies were cross sectional by design. Case-control, cohort studies and experimental studies were few and far between in number. The proportion of clinical trials in health research in India remains very low.(3) This is possibly due to the high resources necessary for carrying out longitudinal and experimental studies. This also highlights a major gap in methodological diversity of health research in the state and needs further evaluation of the causes, capacity building necessary and support systems available for experimental and longitudinal studies.(17-19)

We observed almost an equal number of Community based studies and the hospital-based studies during the decade. While more than two-thirds of the publications comprised of more than three authors, the collaboration between authors was limited to a few groups from specific institutions and there was scarce collaboration between these groups. This possibly implies lack of interdisciplinary collaborations and multicentric studies. Strong professional networking and working groups on specific topics of common interest would be useful in improving collaborative research.

More than two fifth of the publications were based on funding from outside India. This is significant from 2 points of view- one, the quality of proposals and research questions seem to be good enough to get funding from organizations outside India; on the other hand, this also shows a possible lack of access to domestic funding and therefore an over dependence on foreign funds for research. This is counter to the need of setting research priorities from within and calls for an increase in local investments in health research.

The quality of research in India needs to be included in health policy decisions and capacity strengthening by building research institutions of international standards, especially in resource-poor states such as Odisha would be a step in the right direction.(20) 
Evidence from the published body literature from the state of Odisha helps understand the trends and priorities researchers and is necessary for understanding the health research needs of the region. This likewise furnishes us with the knowledge on how the trend of exploration has moved its focus from one research domain to another. This information eventually serves as a foundation for generating better evidence, focussing resources and overall improved evidence-based policy and practice.(21) Lastly, it also helps us to identify the gaps in the disease and health system knowledge which can shape the future research agenda in the region.

The strengths of the study include an exhaustive search in multiple databases with a detailed, explicit and replicable methodology. A major limitation of our study, as with most bibliometric analyses, is that the quality of publications could not be ascertained. The study included only journal articles in English, which is the major language for scientific communication in India and is used almost exclusively in health literature. Health research is a broad and multifaceted topic and although our search strategy was broad, we possibly could have missed tangential topics of interest and acknowledge this limitation. Similarly, a significant proportion of literature from non-indexed journals and grey literature have not been analysed.

\section{Conclusion:}

This bibliometric analysis analyses publications from 2011 to 2020 showing a substantial increase in the number of publications in this decade. This detailed analysis identifies research gaps and helps in generating evidence for future policy implications. Further indepth analysis within individual domains will help in identifying critical areas for improvement and strengthening health system as a whole in Odisha. Priority setting of health care problems, increased funding and capacity building can give a much-necessitated impetus to more quality and evidence-based research for aiding policy implementation and improvement of overall health of Odisha.

\section{$\underline{\text { Acknowledgment: }}$}

We acknowledge PuRI Consortium (Public Health Research Initiative), Bhubaneswar and HIDA Unit (Health Informatics \& Data Analytics), ICMR-RMRC, Bhubaneswar for providing needful resources to complete this research. 


\section{REFERENCES:}

1. Nass SJ, Levit LA, Gostin LO, Rule I of M (US) C on HR and the P of HITHP. The Value, Importance, and Oversight of Health Research. 2009 [cited 2021 Aug 9]; Available from: https://www.ncbi.nlm.nih.gov/books/NBK9571/

2. Dandona L, Sivan YS, Jyothi MN, Bhaskar VU, Dandona R. The lack of public health research output from India. BMC Public Heal 200441 [Internet]. 2004 Nov 25 [cited 2021 Aug 9];4(1):1-12. Available from: https://bmcpublichealth.biomedcentral.com/articles/10.1186/1471-2458-4-55

3. Dandona L, Raban MZ, Guggilla RK, Bhatnagar A, Dandona R. Trends of public health research output from India during 2001-2008. BMC Med 200971 [Internet]. 2009 Oct 14 [cited 2021 Aug 9];7(1):1-13. Available from: https://bmcmedicine.biomedcentral.com/articles/10.1186/1741-7015-7-59

4. Ray S, Shah I, Nundy S. The research output from Indian medical institutions between 2005 and 2014. Curr Med Res Pract. 2016 Mar;6(2):49-58.

5. Aayog N. HEALTHY STATES PROGRESSIVE INDIA Report on the Ranks of States and Union Territories HEALTH INDEX |. 2019 [cited 2021 Aug 9]; Available from: http://social.niti.gov.in/

6. Thomas D, Sarangi BL, Garg A. Closing the health and nutrition gap in Odisha, India: A case study of how transforming the health system is achieving greater equity. Soc Sci Med. 2015 Nov 1;145:154-62.

7. Merigó JM, Núñez A. Influential journals in health research: a bibliometric study. Glob Heal 2016121 [Internet]. 2016 Aug 22 [cited 2021 Jul 17];12(1):1-12. Available from: https://globalizationandhealth.biomedcentral.com/articles/10.1186/s12992-016$0186-4$

8. Improving Health in India - India Research Center [Internet]. [cited 2021 Jul 17]. Available from: https://www.hsph.harvard.edu/india-center/improving-health-in-india/ 
9. ICMR: The Sorry State of Medical Research in India, Health News, ET HealthWorld [Internet]. [cited 2021 Jul 17]. Available from:

https://health.economictimes.indiatimes.com/news/industry/the-sorry-state-of-medicalresearch-in-india/58241623

10. Abstract. An analysis of health status of Orissa in specific reference to health equity.

11. Rayyan - Intelligent Systematic Review [Internet]. [cited 2021 Jul 17]. Available from: https://www.rayyan.ai/

12. Classification of Diseases (ICD) [Internet]. [cited 2021 Jul 17]. Available from: https://www.who.int/standards/classifications/classification-of-diseases

13. Conceptual Framework. SAGE Encycl Educ Res Meas and Evaluation [Internet]. 2018 [cited 2021 Jul 17]; Available from: https://methods.sagepub.com/reference/the-sage-encyclopedia-of-educationalresearch-measurement-and-evaluation/i5552.xml

14. R: The R Project for Statistical Computing [Internet]. [cited 2021 Jul 17]. Available from: https://www.r-project.org/

15. VOSviewer - Visualizing scientific landscapes [Internet]. [cited 2021 Jul 17]. Available from: https://www.vosviewer.com/

16. Keshri VR, Tigga NS. Where there is no research: a bibliometric analysis of health research output from the resource-constrained Bihar state of India. J Glob Heal Reports. 2020 Sep 9;

17. MS S. Methodology Series Module 3: Cross-sectional Studies. Indian J Dermatol [Internet]. 2016 May 1 [cited 2021 Aug 9];61(3):261-4. Available from: https://pubmed.ncbi.nlm.nih.gov/27293245/

18. S D. Clinical trials and healthcare needs in India: A difficult balancing act but opportunities abound! Perspect Clin Res [Internet]. 2017 Oct 1 [cited 2021 Aug 9];8(4):159-61. Available from: https://pubmed.ncbi.nlm.nih.gov/29109931/ 
19. Burt T, Sharma P, Dhillon S, Manchanda M, Mittal S, Trehan N. Clinical Research Environment in India: Challenges and Proposed Solutions. J Clin Res Bioeth [Internet]. 2014 [cited 2021 Aug 9];5(6):1. Available from:

/pmc/articles/PMC4290669/

20. Kalita A, Sachin S, Patel V. Public health research in India in the new millennium: A bibliometric analysis. Glob Health Action. 2015;8(1).

21. Poletto V, Faraco Junior, Italo Medeiros. Bibliometric study of articles published in a Brazilian journal of pediatric dentistry. Braz Oral Res. 2010 Jan;24(1):83-8. 